\title{
El papel de la normativa urbana en el desarrollo turístico y la protección del ambiente
}

\author{
Roy Allan Jiménez Céspedes
}

Invitado nacional

Ministerio de Vivienda y Asentamientos Humanos

allan.delalto@gmail.com

Recibido: 26 de junio del 2016

Aceptado: 31 de marzo del 2017

\section{Roy Allan Jiménez Céspedes}

Universidad de Alcalá. Máster Universitario en Proyecto Avanzado de Arquitectura y Ciudad de la Universidad de Alcalá, con Énfasis en Intervenciones Urbanas.

\begin{abstract}
Resumen:
La región Chorotega en el pacífico costarricense, ha recibido una fuerte inversión por parte del sector turismo. Actualmente, procesos como el impacto del turismo residencial, la disponibilidad de recurso hídrico y la ocupación de áreas ambientalmente frágiles preocupan a diferentes sectores del gobierno y de la sociedad civil. Este es un fenómeno multicausal que abarca, desde la falta de planificación territorial hasta la escasez de recursos para los procesos de tramitación y control. La poca participación de las comunidades en la planificación y ejecución de proyectos son otros de los factores relacionados.

En Costa Rica, se está cuestionando la efectividad de la normativa para la conservación de los recursos naturales frente al desarrollo turístico. El extenso marco normativo para la regulación de las actividades turísticas presenta una serie de inconsistencias que facilitan la ocupación y sobre explotación de áreas ambientalmente frágiles.
\end{abstract}

Palabras Clave: desarrollo urbano; normativa; recursos naturales; turismo.

The role of urban regulation in touristic development and in environmental protection

\begin{abstract}
:
The Chorotega region, in the pacific coast, has received strong investments from touristic industry since the $90 \mathrm{~s}$. Right now, the impact of residential tourism, the integrity for water sources, and the construction activities on fragile areas are the main concerns from government and population groups. The lack of land use planning, the difficulties on the implementation of a very complex legal framework and the weakness of local authorities are some of the causes. Additionally, the scarce participation of local population in planning and implementation of projects are also important components of the problem.

In Costa Rica, the legal frame has been questioned on its effectiveness to guarantee the conservation of natural resources against the impact of tourism industry. The regulations on touristic activities expose several inconsistencies that facilitates the occupation of fragile zones and endanger natural resources.
\end{abstract}

Keywords: urban development; regulations; natural resources; tourism. 


\section{Introducción}

$1 \quad$ El artículo 50 de la Constitución política de Costa Rica garantiza un ambiente sano y ecológicamente equilibrado para los ciudadanos.

$2 \quad$ El Programa Estado de la Nación es un programa institucional continuo de investigación y forma parte del Consejo Nacional de Rectores de las Universidades Públicas de Costa Rica.
I impacto de los desarrollos turísticos de gran escala sobre los recursos naturales en Costa Rica es un fenómeno complejo y multicausal. En primera instancia, la normativa para la regulación de la actividad turística se ubica en el centro del debate sobre su efectividad en la defensa de un ambiente sano y ecológicamente equilibrado1.

La aparición de conflictos socioambientales por el acceso prioritario a recursos naturales ha provocado, en algunos casos, posiciones contradictorias del Estado costarricense. En este particular, algunos estudios realizados han analizado la coherencia entre las políticas ambientales del Estado y la apuesta por la atracción de Inversión extranjera directa, encontrando posibles problemas de dispersión normativa e irregularidades en los mecanismos de control (Barrantes Reynolds, 2011). Adicionalmente, el Programa del Estado de la Nación ${ }^{2}$ ha alertado, desde el 2006, sobre los impactos acumulativos de la actividad turística de gran escala en los recursos naturales de la región Chorotega y el Pacífico Central (Román, 2006).

Las principales preocupaciones por el impacto del turismo de gran escala están asociadas a los efectos del turismo residencial, el manejo del recurso hídrico, los grandes movimientos de tierra, cambios en el uso del suelo amparado en figuras como la parcelación agrícola, y la producción y manejo de los residuos sólidos. (Román, 2006). [figura 1]

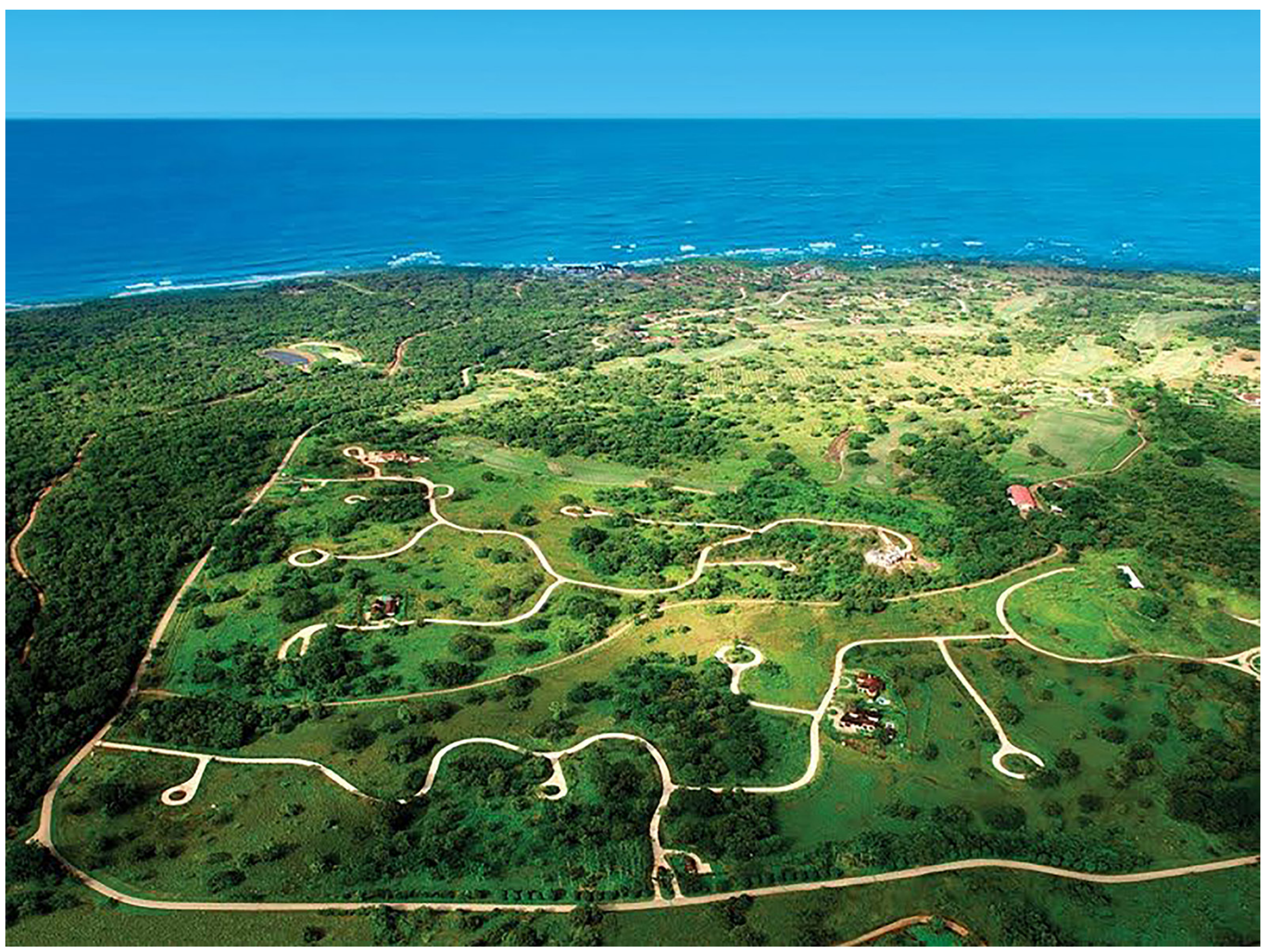

A

Figura 1. El impacto del turismo residencial:

El impacto del Turismo residencial es una de

las principales preocupaciones. Estos grandes conjuntos se convierten en comunidades

cerradas dirigidas primordialmente al mercado

extranjero. Imagen con fines ilustrativos, fuente:

$\mathrm{http} / / /$ summercoastrealty.net/properties/lasgolondrinas-12/ 
Figura 2. Ubicación de la región Chorotega. Imagen de Elaboración propia.
Desde la década de 1980, el desarrollo turístico en Costa Rica se ha consolidado como la principal fuente de divisas del país. Según el Consejo Mundial de Viajes y Turismo, el aporte directo del turismo al Producto Interno Bruto costarricense ascendió al 4,8\% en el 2015 y alcanza el $12,6 \%$ en su contribución total. Casi tres puntos sobre el $9,8 \%$ de media a nivel mundial.

El turismo masivo, con fuerte componente inmobiliario, empezó en la región Chorotega y en la provincia de Guanacaste hacia 1990. La cercanía del país con Estados Unidos y Canadá, así como sus favorables condiciones climáticas y topográficas, han jugado un papel relevante en la atracción de inversión extranjera proveniente de la industria turística (Navas, Cuvi, 2015). La apertura en 2002 del Aeropuerto Internacional Daniel Oduber en el cantón de Liberia ha consolidado el crecimiento de la actividad turística en la Región. [figura 2]

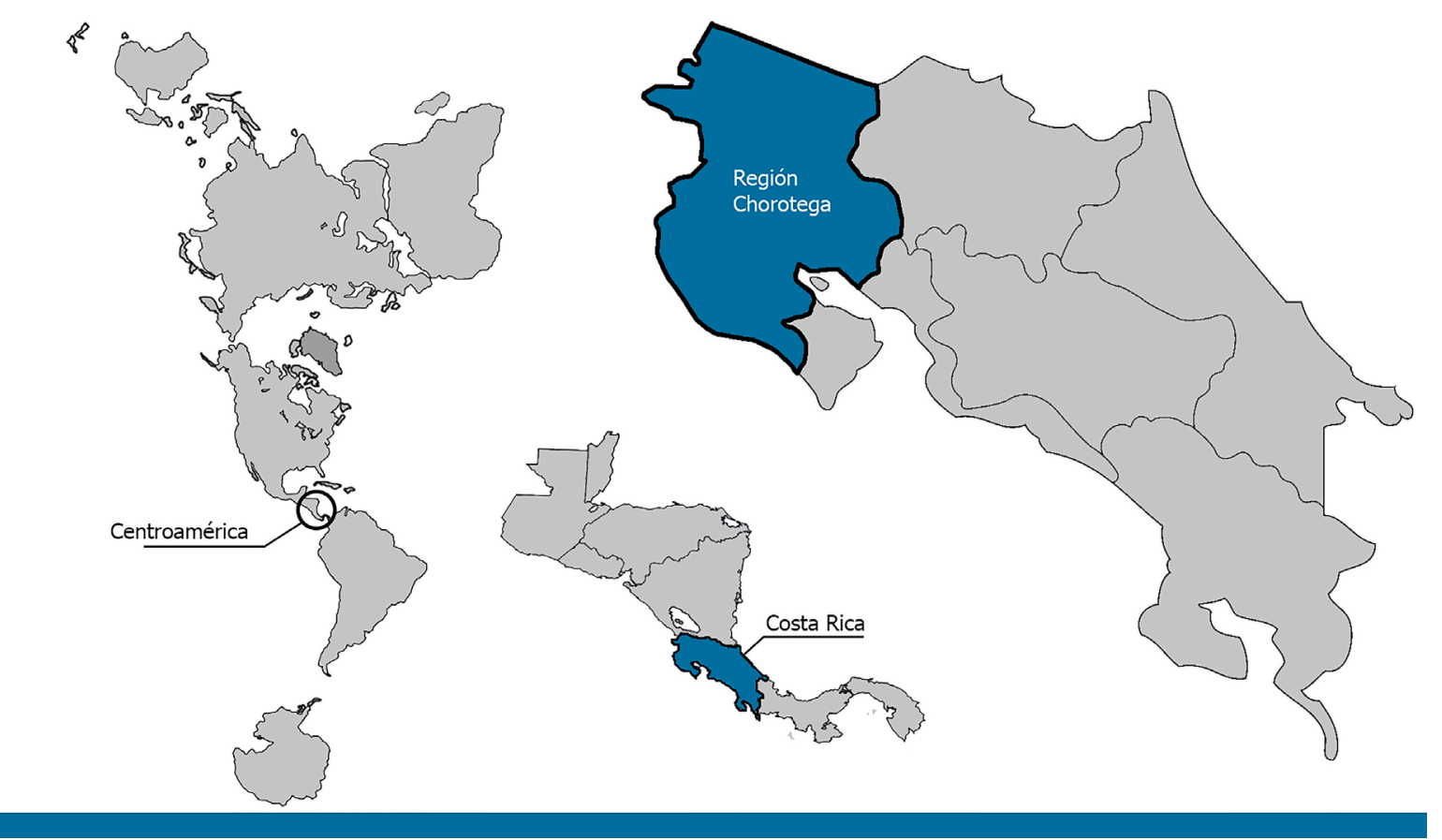

En la región Chorotega se han generado una serie de conflictos entre comunidades locales y algunos actores del sector turismo. Los que más llaman la atención son los relacionados con el recurso hídrico, pues la demanda de agua se ha visto bajo una fuerte presión por parte de consorcios hoteleros que desde la década de los noventa han invertido fuertemente en la región (Tribunal Centroamericano del Agua, 2014). Otro tema que ha generado la reacción de diversos grupos ecologistas es el comportamiento del turismo masivo ante procesos naturales como el desove de las tortugas.

Estos conflictos se ven maximizados por los frecuentes períodos de sequía y escasez de agua que caracterizan a la región y que están muy asociados a los fenómenos climáticos de El Niño y La Niña.

En el presente artículo se discute la congruencia de la normativa encargada de regular la actividad turística con la protección de los recursos naturales. Para esto se realiza el análisis de casos específicos. Como punto de partida, se considera que la normativa y el marco institucional presentan una serie de condiciones que permiten la ocupación de zonas frágiles y dificultan la conservación de los recursos naturales.

Para desarrollar estas ideas se ha utilizado la siguiente metodología:

En una primera instancia se estudió la normativa para la regulación de las actividades turísticas, haciendo énfasis en la planificación urbana. Se omitieron leyes específicamente aprobadas para el desarrollo de polos turísticos determinados y se concentró el estudio en las normativas locales y nacionales vigentes. La normativa asociada a las Áreas Silvestres Protegidas -ASP- no se incluye en el estudio, por ser su planificación y gestión responsabilidad del Ministerio de Ambiente.

Se analizó la aplicación real de la normativa para la regulación de las actividades turísticas en función de su efectividad para garantizar la conservación de los recursos naturales de los cuales hace uso.

Porúltimo, las conclusiones del artículo pretenden: a) dar una luz sobre las inconsistencias y desarticulaciones presentes en la normativa y b) definir los puntos a considerar en un eventual proceso de actualización normativa en el tema urbano territorial asociado a la actividad turística. 


\section{Sobre la normativa para la regulación de la actividad turística y la protección de los recursos naturales}

Para efectos del análisis, la normativa se dividirá en tres grandes grupos o "generaciones":

Normativa de planificación urbanística y regulación de la construcción.

Normativa del sector ambiente y conservación de recursos naturales, que se relaciona con los procesos urbanos.

Normativa del sector turismo, que promueve un tipo determinado de planificación, 0 bien, de protección de recursos naturales. Esta categorización de la normativa se realiza considerando el año de aprobación de las diferentes normas [figura 3] y la naturaleza de los objetivos perseguidos.

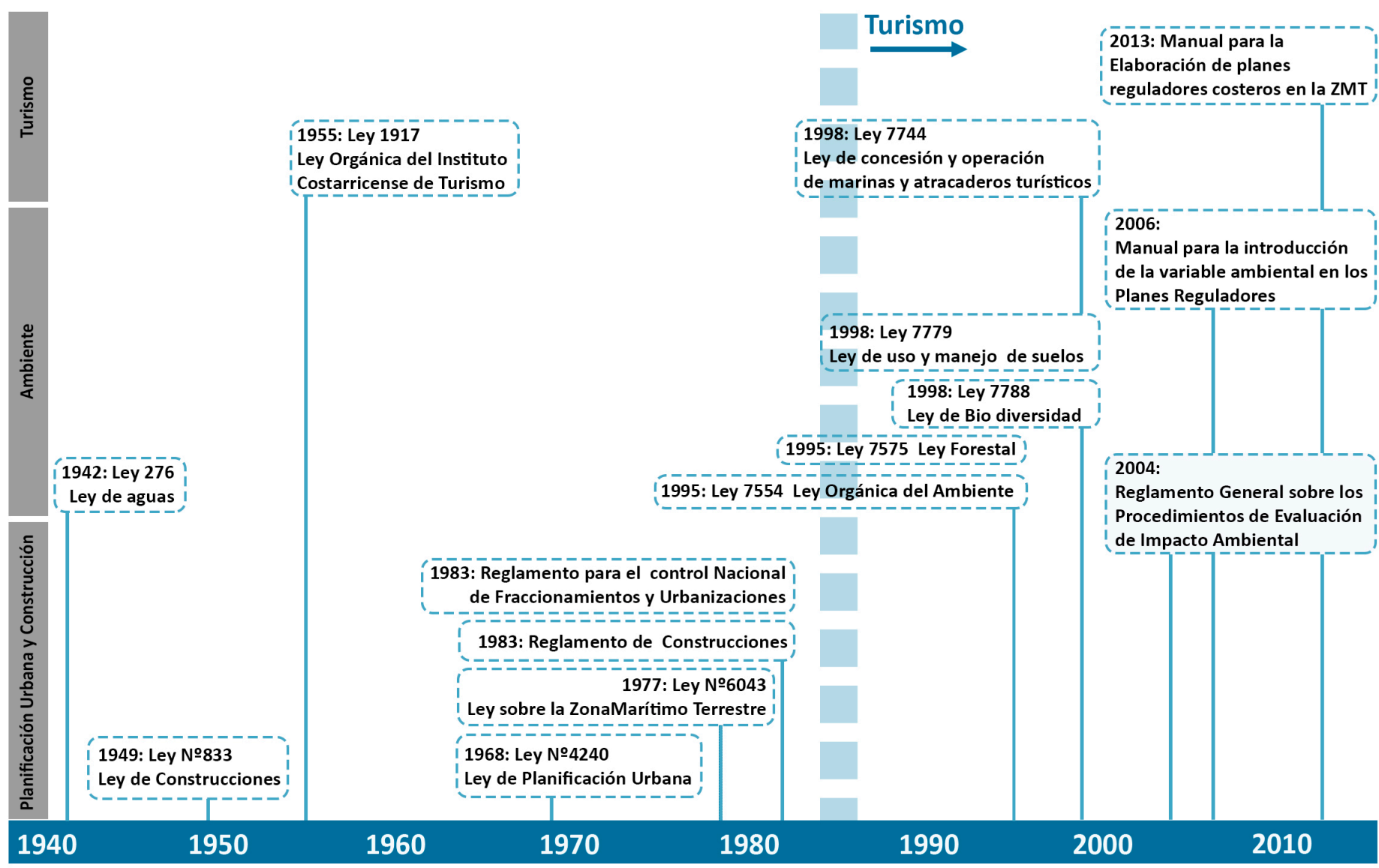

Línea del tiempo normativa

Se consignan los instrumentos legales que determinan procedimientos de tramitación o bien parámetros urbanos

Figura 3. Línea del tiempo normativa. Imagen de elaboración propia con datos del Sistema Costarricense de Información Jurídica de la Procuraduría General de la República.
La normativa asociada a la planificación urbanística encuentra su principal cuerpo legal en la Ley de Planificación Urbana de 1968 -LPU- y la Ley sobre la Zona Marítimo Terrestre de 1977 -LZMT-. Ambas leyes introducen la figura del plan regulador como el instrumento normativo por excelencia y reconocen la autonomía del régimen municipal para planificar y controlar el desarrollo de sus territorios.

"Aun cuando la legislación asociada al tema de la planificación data de los cuarentas, es notorio que en ese momento se incorporaban herramientas básicas para impulsar una política en los usos del territorio nacional." (Cabrera \& Sánchez, 2009, p.50).

Mientras la LPU determina la obligatoriedad de los municipios de contar con un plan regulador, la LZMT establece este mismo instrumento de una manera más específica para todas las zonas litorales hasta $200 \mathrm{~m}$ desde la línea de pleamar. Esta franja se denomina zona marítimo terrestre y se divide en la zona pública -50 metros desde la línea de pleamar- y la zona restringida -los 150 metros restantes-. La zona restringida es susceptible a ser concesionada, previa aprobación del plan regulador costero respectivo.

La diferencia sustancial entre ambos casos radica en la propiedad del suelo que regulan y en su alcance. Los planes reguladores cantonales establecen los parámetros para el correcto desarrollo urbano en toda la jurisdicción municipal. Por otra parte, los planes reguladores costeros se enfocan únicamente en la franja litoral (de propiedad pública) y debe ser la herramienta para regular el otorgamiento de concesiones en zona marítimo terrestre. Las municipalidades deben seguir los lineamientos establecidos en los planes reguladores para la emisión de las licencias de construcción o bien las concesiones en la zona marítimo terrestre. 
Figura 4. Planificación turística en la Región Chorotega. Cantones de la provincia de Guanacaste y Unidades de Planificación Turística definidas por el Instituto Costarricense de Turismo. Imagen de elaboración propia con datos del Instituto Costarricense de Turismo.
Los planes reguladores, tal y como han sido definidos por la legislación, determinan la ordenación de todo el territorio. Todo el suelo ha de pertenecer a alguno de los regímenes de suelo previstos y estar incluido en alguna clasificación de suelo que establezca con diverso grado de precisión su destino y sus incompatibilidades. (Maxera, 2008, p.192)

Otra diferencia importante es que los planes reguladores costeros deben seguir los lineamientos y políticas turísticas establecidas por el Instituto Costarricense de Turismo -ICT- a través del Plan Nacional de Turismo Sostenible. Del mismo modo, el Plan Nacional de Turismo Sostenible debe guardar coherencia con el Plan Nacional del Desarrollo Urbano -potestad del Instituto Nacional de Vivienda y Urbanismo- y con el Plan Nacional de Desarrollo -competencia del Ministerio de Planificación Nacional(Cabrera \& Sánchez, 2009). El Plan Nacional de Turismo Sostenible, establece una serie de unidades de planificación turística en estrecha relación con las áreas silvestres protegidas y las playas de la región Chorotega [figura 4]. Estas unidades de planificación obedecen a una división territorial diferente a la establecida para los 11 cantones que componen la provincia de Guanacaste.
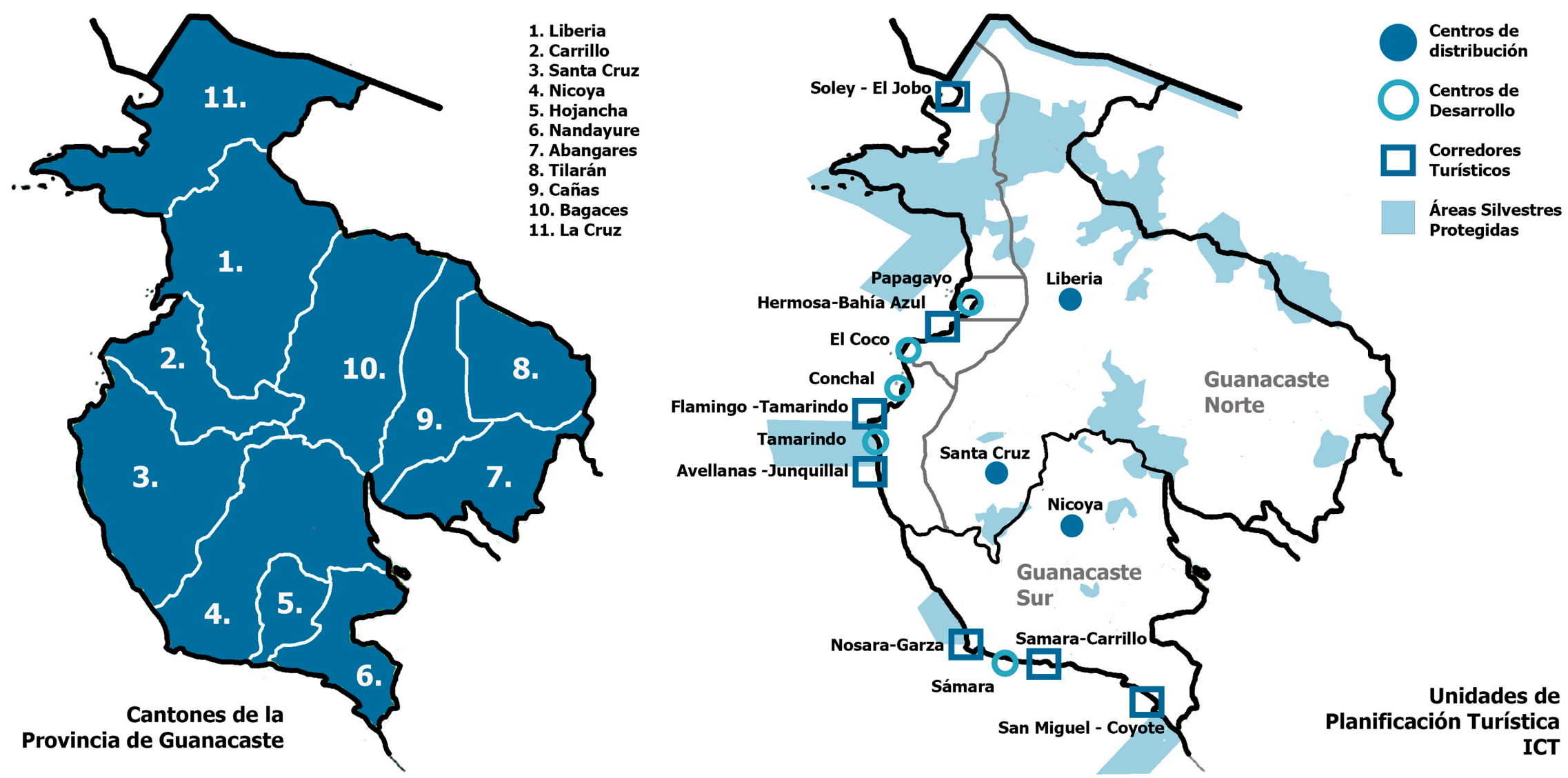

Así pues, un municipio con zonas litorales debe contar con su plan regulador cantonal y con los planes reguladores costeros que sean necesarios. En función de esta planificación territorial, la municipalidad debe estudiar y otorgar los permisos para toda construcción.

La normativa asociada al sector ambiente tiene su principal estructura principal en la Ley Orgánica del Ambiente -LOA- de 1995. La LOA introduce dos factores de cambio importantes en la planificación y la tramitación de los proyectos inmobiliarios: El concepto del ordenamiento territorial y la creación de la Secretaría Técnica Ambiental (SETENA). De esta manera, tanto la tramitación de los planes reguladores como la aprobación de proyectos constructivos, deben incluir un análisis de la variable ambiental, el cual es revisado por la SETENA. Los planes reguladores cantonales y costeros deben incluir un análisis para la introducción de la variable ambiental que debe ser aprobado por la SETENA según lo establecido en el decreto 32967.

Las actividades humanas que alteren o destruyan elementos del ambiente o generen residuos, materiales tóxicos o peligrosos, requerirán una evaluación de impacto ambiental por parte de la Secretaría Técnica Nacional Ambiental creada en esta ley. Su aprobación previa, de parte de este organismo, será requisito indispensable para iniciar las actividades, obras o proyectos. Las leyes y los reglamentos indicarán cuales actividades, obras o proyectos requerirán la evaluación de impacto ambiental. (Ley $\mathrm{N}^{\circ}$ 7554, Art. 17) 
El artículo 1 del Decreto 32967 indica:

En toda planificación de uso de suelo que se desarrolle en el país, incluyendo los planes reguladores cantonales o locales, públicos o privados, en los que se planifique el desarrollo de actividades, obras o proyectos que pudiesen generar efectos en el ambiente, deberá integrarse la variable ambiental de acuerdo con el Procedimiento para la Introducción de la variable ambiental en los Planes Reguladores u otra Planificación de uso del suelo.

A continuación de la LOA, otras leyes han sido aprobadas en el marco de las convenciones internacionales suscritas por Costa Rica y producto de la Cumbre de la Tierra, realizada en Río de Janeiro en 1992.

De esta forma, se suman al marco legal la Ley Forestal -aprobada en 1995 y en consonancia con el Convenio Marco de Naciones Unidas sobre el Cambio Climático-, la Ley de Biodiversidad -aprobada en 1998 y en consonancia con el Convenio sobre la Diversidad Biológica- y la Ley de Uso y Manejo de Suelos -aprobada en 1998 y en consonancia con la Convención de las Naciones Unidas de Lucha Contra la Desertificación-.

Con la entrada en vigencia de este nuevo cuerpo normativo, se agregan actores y métodos de fiscalización de las actividades inmobiliarias, incluidas por supuesto las actividades turísticas de gran escala. En el próximo capítulo se analiza la efectividad de estos instrumentos en la conservación de los enclaves naturales que el turismo explota como recursos turísticos.

Desde el sector turismo, el marco normativo no establece mayores requisitos desde el punto de vista de la conservación ambiental, delegando esta labor a los entes competentes según la normativa. Sin embargo, y como una manera de establecer un modelo de desarrollo para la zona marítimo terrestre, desde 2013 el Instituto Costarricense de Turismo estableció el manual para la elaboración de Planes reguladores costeros en la zona marítimo terrestre que regula e intenta controlar el uso intensivo de esta franja de tierra. Sin embargo, algunas municipalidades acusan que esta normativa restringe el desarrollo del cantón y busca intensificar el uso turístico en las zonas aledañas no reguladas por el ICT.

Es posible identificar un marco normativo diverso en lo que respecta a la regulación de las actividades turísticas. Varios autores han señalado la heterogeneidad de la normativa como un problema que no permite un adecuado control y fiscalización de las actividades inmobiliarias.

La legislación costarricense se rige por un sistema regulatorio que comprende una amplia gama de leyes y decretos por materias específicas, los cuales se someten a la interpretación de los Tribunales de Justicia, la Procuraduría, la Contraloría y dependencias administrativas que operan y resuelven conforme a la disposición en la normativa legal vigente. Leyes que interactúan con fuerza en materia pública y administrativa se acompañan por una serie de decretos que regulan y amplían los efectos de los contenidos de la ley. Integrar las leyes y los decretos promulgados es una de las tareas más exhaustas para un operador del derecho que toma decisiones sobre los aspectos regulatorios, considerando la cantidad de decretos y las competencias de diferentes instituciones sobre un tema en particular, las cuales no están exentas de duplicidad, traslapes y conflictos funcionales. (Cabrera \& Sánchez, 2009, p. 5)

El marco legal asociado a la regulación de la actividad turística está compuesto por diferentes ramas, no precisamente articuladas entre sí. La normativa sufre de inconsistencias, tanto conceptuales como institucionales. Adicionalmente, se encuentra apoyado sobre gobiernos locales, en su mayoría sin las herramientas técnicas ni recursos humanos para garantizar el cumplimiento de los procedimientos legales (Honey, Vargas \& Durham, 2010).

El ordenamiento territorial adolece del mismo problema. El desarrollo urbano local se ve afectado por la dispersión de los diferentes cuerpos normativos y la falta de coherencia entre las diferentes leyes (Román, 2006). Esto inhibe y retrasa la aprobación de los planes reguladores de los diferentes municipios. 


\section{Aplicación de la normativa y mecanismos de control parala protección de los recursos naturales}

\section{Tramitación y control}

El cumplimiento de los parámetros de planificación territorial, los diferentes procedimientos de tramitación para la construcción y los mecanismos posteriores de control, son aspectos estratégicos en la regulación de cualquier actividad inmobiliaria.

La tramitación tiene el objetivo de hacer cumplir el marco legal (y sus objetivos ulteriores) en el desarrollo de las actividades relacionadas con el proceso constructivo. Tramitar implica cumplir con una serie de requisitos normativos solicitados por diferentes instancias de control, con el objetivo de ejercer actividades específicas en terrenos 0 zonas de aptitud turística (Cabrera \& Sánchez, 2009). Por lo tanto, se considera que la tramitación es la primera instancia de control entre el enclave turístico de gran escala y la integridad de los recursos naturales.

Los mecanismos de control aparecen como el brazo operativo de la norma para garantizar el cumplimiento de la ley. Tanto la tramitación como el control juegan un papel importante en la tutela de los recursos naturales.

La Ley de Construcciones -Ley \#833 de 1949-, en su Art. 74 otorga a las municipalidades la potestad para el otorgamiento de las licencias constructivas: "Toda obra relacionada con la construcción, que se ejecute en las poblaciones de la República, sea de carácter permanente o provisional, deberá ejecutarse con licencia de la Municipalidad correspondiente". Como se mencionó en el capítulo anterior, estas licencias constructivas deben cumplir con lo establecido en los planes reguladores respectivos. Queda fuera de la jurisdicción de los gobiernos locales lo relacionado con la delimitación y visado en áreas silvestres protegidas. La administración de estas zonas recae en el Ministerio de Ambiente (Cabrera \& Sánchez, 2009).

Esta perspectiva nos presenta dos escenarios: Cantones que cuentan con plan regulador y los que no cuentan con este instrumento. De no existir plan regulador, las municipalidades deben seguir lo establecido en los cuerpos normativos de escala nacional. Estas municipalidades se enfrentan a una realidad aún más compleja, debido a que no cuentan con un régimen normativo de clasificación pormenorizado del suelo y deben tener en cuenta para la aprobación de los proyectos, insumos técnicos y normativos que, en muchos casos, no se encuentran a escala local. Este es el caso de la Ley de Planificación Urbana o el Reglamento para el Control Nacional de Fraccionamientos y Urbanizaciones, normas nacionales de carácter general que, en el contexto del ejercicio de control municipal, no cuentan con ninguna consideración del contexto de las diferentes localidades.

El instrumento de planificación más común son los planes reguladores, pero no significa que han sido el modelo para un ordenamiento territorial costero.

Gran parte de este territorio adolece de esta herramienta, a veces hay a veces no, parciales difícilmente totales, no son cantonales sino circunscritos a un área de interés para desarrollar. La CGR en informes emitidos entre el año 2006 y 2007 informó de la carencia de planes reguladores en importantes cantones costeros, entre ellos Santa Cruz en Guanacaste y Aguirre en Puntarenas.

Al mismo tiempo, reportó casos de planes reguladores que se aprobaron en áreas boscosas propiedad del Patrimonio Natural del Estado (Playa Pará en Aguirre de Puntarenas y Punta Castilla en la Cruz de Guanacaste) (Cabrera \& Sánchez, 2009, p.53)

La ausencia total de planes reguladores cantonales, o bien, la existencia de planes reguladores sin variable ambiental incluida, representa un primer vacío que promueve el impacto negativo de los desarrollos turísticos de gran escala. Sin la presencia de estos instrumentos, las municipalidades no pueden establecer un marco normativo específico para las actividades que pretendan promover y controlar. Por lo tanto, el gobierno local no puede establecer el tamaño, localización y tipo de actividad turística, pero sí deberá tramitar los permisos de construcción. 
En la figura 5 se puede apreciar el estado de los planes reguladores en la provincia de Guanacaste. De los 11 municipios que componen la provincia, únicamente 5 cuentan con plan regulador, pero en todos los casos el alcance territorial de estos instrumentos se limita al distrito central del cantón -específicamente la trama urbana- y ninguno cuenta con variable ambiental aprobada.
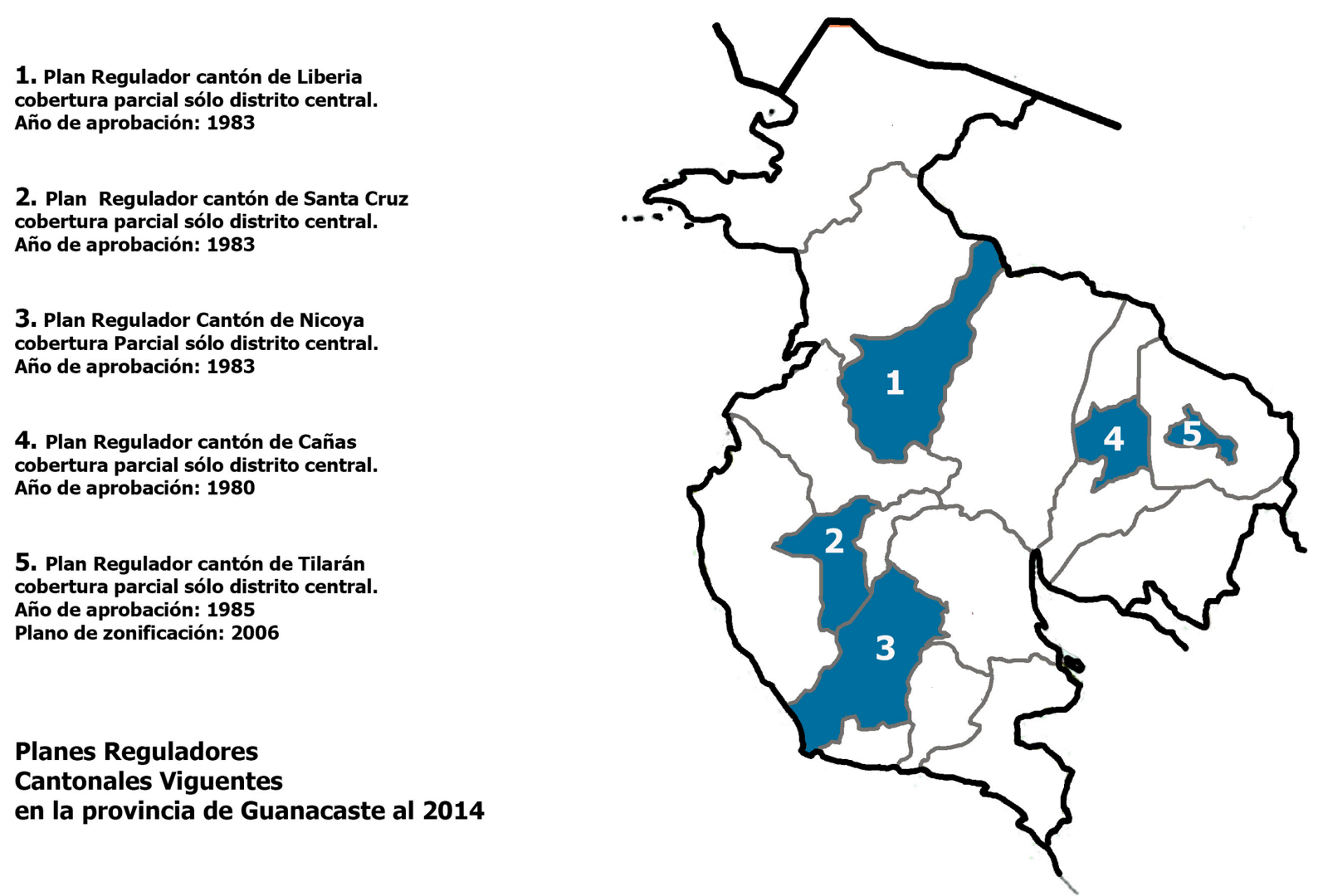

Figura 5. Planes Reguladores vigentes en la provincia de Guanacaste al año 2014. Imagen de elaboración propia con datos de Instituto Nacional de Vivienda y Urbanismo.
Adicionalmente, los planes reguladores vigentes muestran fechas de aprobación que datan de la década de los ochentas, habiendo experimentado muy pocos procesos de actualización. Es decir, la poca planificación urbanística presente en la zona responde a un período muy anterior a la llegada de la industria turística. Al no contar ninguno de estos planes con variable ambiental, carecen de un enfoque que tome en cuenta los procesos naturales de escala cantonal y regional.

En las zonas costeras encontramos la presencia de mayor cantidad de planes reguladores costeros. Sin embargo, como se explicó, estos planes son de un alcance muy limitado. Otro aspecto que influye directamente en la poca incidencia de los planes reguladores costeros es la escasa participación ciudadana. Esto genera que, en muchas ocasiones, las expectativas y necesidades de la población local terminen invisibilizadas. (Maxera, 2008).

De esta manera, con una gran parte del territorio sin regulación territorial local, las municipalidades deben tramitar los proyectos turísticos con los requisitos estipulados en las leyes de carácter nacional. Esto significa que el proceso de tramitación obedece a un marco legal que contiene leyes de toda índole, con fechas de aprobación muy dispares y, en algunos casos, alejadas de la realidad de las comunidades.

La tramitación municipal de proyectos es un complejo compendio de requisitos establecidos por diferentes leyes. Estos requisitos incluyen aspectos de toda índole que pasan por la presentación de los documentos registrales de la propiedad, alineamientos a zonas de protección o ríos, alturas máximas en relación a aeropuertos y campos de aterrizaje y la disponibilidad de servicios básicos para abastecer el desarrollo planteado.

Adicionalmente, el trámite previo ante la Secretaría Técnica Ambiental es requisito indispensable y su complejidad varía en función de la escala del desarrollo. Sin embargo, atender la gran de demanda de solicitudes para construir desarrollos turísticos pone a los departamentos municipales, encargados de otorgar los permisos, en situación de desventaja. Aunado a esto, la ausencia de planes reguladores con viabilidad ambiental aprobada según lo establecido en el Decreto 32967, impide el análisis del impacto global de los desarrollos inmobiliarios. La cantidad, dimensiones y montos de inversión que representan estos desarrollos, sobrepasan las capacidades de las municipalidades ante las condiciones de falta de recursos para planificación y control (Cabrera \& Sánchez, 2009).

La función de control sobre lo establecido en los permisos y licencias constructivas recae directamente en los gobiernos locales. En consecuencia, la debilidad estructural 
presente en los gobiernos locales impide que los mecanismos de control sean constantes y eficaces.

\section{Aplicación de la normativa: algunos vacíos y debilidades.}

Recientemente han sido identificadas irregularidades en la aprobación de algunos planes reguladores costeros que ponen en riesgo recursos naturales de importancia turística como manglares, bosques y patrimonio natural del estado.

Uno de las principales preocupaciones, en relación al impacto de la actividad turística, está relacionado con el turismo residencial. En este formato de actividad, grandes porciones de terreno son utilizadas para el establecimiento de enclaves residenciales en zonas de gran valor ambiental y turístico. Este tipo de desarrollo llama la atención por la gran cantidad de movimiento de tierra que representa.

En relación a este particular, la figura de la parcelación agrícola está siendo utilizada por promotores inmobiliarios para evadir procesos de tramitación y crear un producto inmobiliario a la medida del mercado estadounidense. En principio, la parcelación agrícola consiste en la subdivisión de fincas en parcelas cuya dimensión se encuentre entre 1 y 5 hectáreas para fines agrícolas [figura 6]. Esta figura de segregación está exenta de una gran cantidad de trámites e impuestos, siendo necesario únicamente el visto bueno municipal. Anteriormente se solicitaba, como requisito, la aprobación de la aptitud agrícola de los terrenos por parte de Ministerio de Agricultura y Ganadería, pero este requisito fue suprimido en la modificación al Artículo II.2.1.5 del Reglamento Nacional de Fraccionamientos y Urbanizaciones por acuerdo de la Junta Directiva del Instituto Nacional de Vivienda y Urbanismo, en sesión $\mathrm{N}^{\circ} 5281$ del 28 de mayo del 2003, y publicado en el diario oficial la Gaceta el 5 de junio del mismo año. En esta modificación, también se disminuyó el tamaño de la parcela mínima productiva a 5000 metros cuadrados y aumentó el área construible de la misma.

Este vacío normativo en la tramitación ha dado pie al desarrollo de grandes extensiones de suelo para la construcción de condominios y grandes complejos residenciales. La oferta inmobiliaria asociada ofrece terrenos de 5000 metros cuadrados o más, cercanos a las playas o en zonas de montaña. Esta problemática de urbanización irregular encierra una gran preocupación dado que para la parcelación agrícola no se exige ningún

Figura 6. El problema de la parcelación agrícola. $Y$ Aunque la parcelación agrícola es una figura que busca el aprovechamiento de los suelos con potencial agrícola o forestal, la débil regulación y la carencia de control ha permitido que se utilice para la urbanización irregular de terrenos de grandes extensiones. Imagen de elaboración propia. requisito relacionado con el posible impacto ambiental. Se pone en evidencia que las municipalidades no están preparadas para controlar y proteger las zonas de fragilidad ambiental, o bien, para velar por el cumplimiento de los permisos de construcción (Cabrera, Sánchez, 2009). El uso de parcelación agrícola para la urbanización irregular es un fenómeno que no es exclusivo de la región Chorotega, también se han detectado casos de condominios igualmente tramitados en toda la costa pacífica:

Regulación permisiva y carencia de control
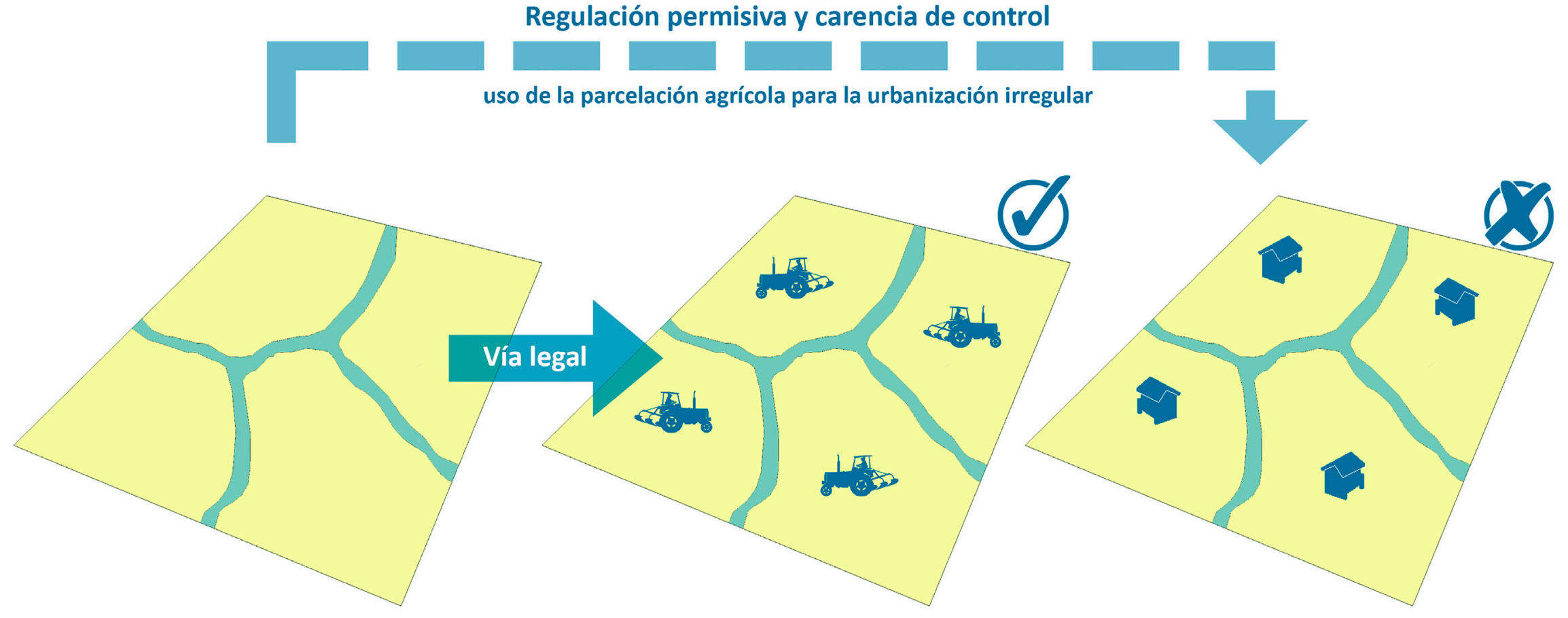

Suelos de interés agrícola o forestal
Parcelación agrícola (Uso agrícola o forestal)
Proyectos inmobiliarios dirigidos al turismo residencial 
El impacto de esta práctica reciente en el litoral pacífico ha sido preocupante en Osa. En el 2007 las denuncias se profundizaron por los movimientos de tierra autorizados por la Municipalidad de Osa en la Fila Costeña, donde las acciones de los desarrolladores preocupan a diferentes sectores. (Cabrera \& Sánchez, 2009, p. 123)

Otro aspecto importante es la interpretación de la normativa de escala nacional. Con el objetivo de poder abastecer los desarrollos turísticos de recurso hídrico (no solo para consumo sino también para piscinas, campos de golf y atracciones similares) los promotores turísticos se han amparado en el Art. 38 de la Ley de Planificación Urbana (4240), el cual permite la inversión de capital privado para la construcción de obra pública:

Los proyectos de urbanización ubicados dentro o fuera del área zonificada que tengan los servicios de acueductos, alcantarillado sanitario y electricidad alejados de sus linderos, deber ser aceptados para su análisis por la Municipalidad y la Dirección de Urbanismo, si el urbanizador se compromete a costear las obras ejecutadas fuera de su propiedad, para ofrecer todos los servicios necesarios.

En este caso, todos los proyectos futuros de urbanización que intenten usar los servicios citados en el párrafo anterior en el período de cinco años, contados desde la terminación de esas construcciones, abonarían al urbanizador una cantidad por cada unidad de vivienda que contenga el nuevo proyecto. La cantidad a abonar será determinada por la institución que tenga a su cargo el servicio correspondiente y se cubrirá al hacerse la conexión física de cada unidad de vivienda.

Una interpretación laxa de este artículo se empleó para construir diversos proyectos Turísticos en la región Chorotega que desataron una fuerte disputa por el agua proveniente del acuífero Sardinal. En 2006, la Municipalidad de Carrillo (carente de plan regulador), autorizó la construcción de diversas infraestructuras turísticas (hoteles, restaurantes, condominios residenciales...) que precisaban de abastecimiento de agua. Para lograrlo, el Instituto Costarricense de Acueductos y Alcantarillados y el inversionista inmobiliario Coco Water S.A. firmaron una carta de entendimiento en la cual Coco Water S.A. construiría el acueducto de $14 \mathrm{~km}$, necesario para llevar el agua a las zonas de interés turístico [figura 7]. El agua sería extraída del acuífero Sardinal (Navas \& Cuvi, 2015).

Ante la cantidad de agua que iba a ser extraída del acuífero (176 lt/seg de 3 perforaciones) y la nula consulta e información a las comunidades, se desató una disputa legal entre las asociaciones comunales, el desarrollador turístico y la Municipalidad de Carrillo.

En 2010, la Sala Constitucional emitió la resolución № 2009-000262 en la cual falla a favor de la Asociación de Pobladores que defendían el agua, estableciendo que las obras debían detenerse hasta determinar la capacidad real del acuífero y realizar un debido proceso de consulta con las poblaciones afectadas (Navas \& Cuvi, 2015).

Figura 7. Acuífero Sardinal, una disputa socio ambiental. Detalle de las obras realizadas al amparo del Art. 38 de la Ley de Planificación urbana en el acuífero Sardinal. Imagen de elaboración propia son base en esquema del periódico La Nación.

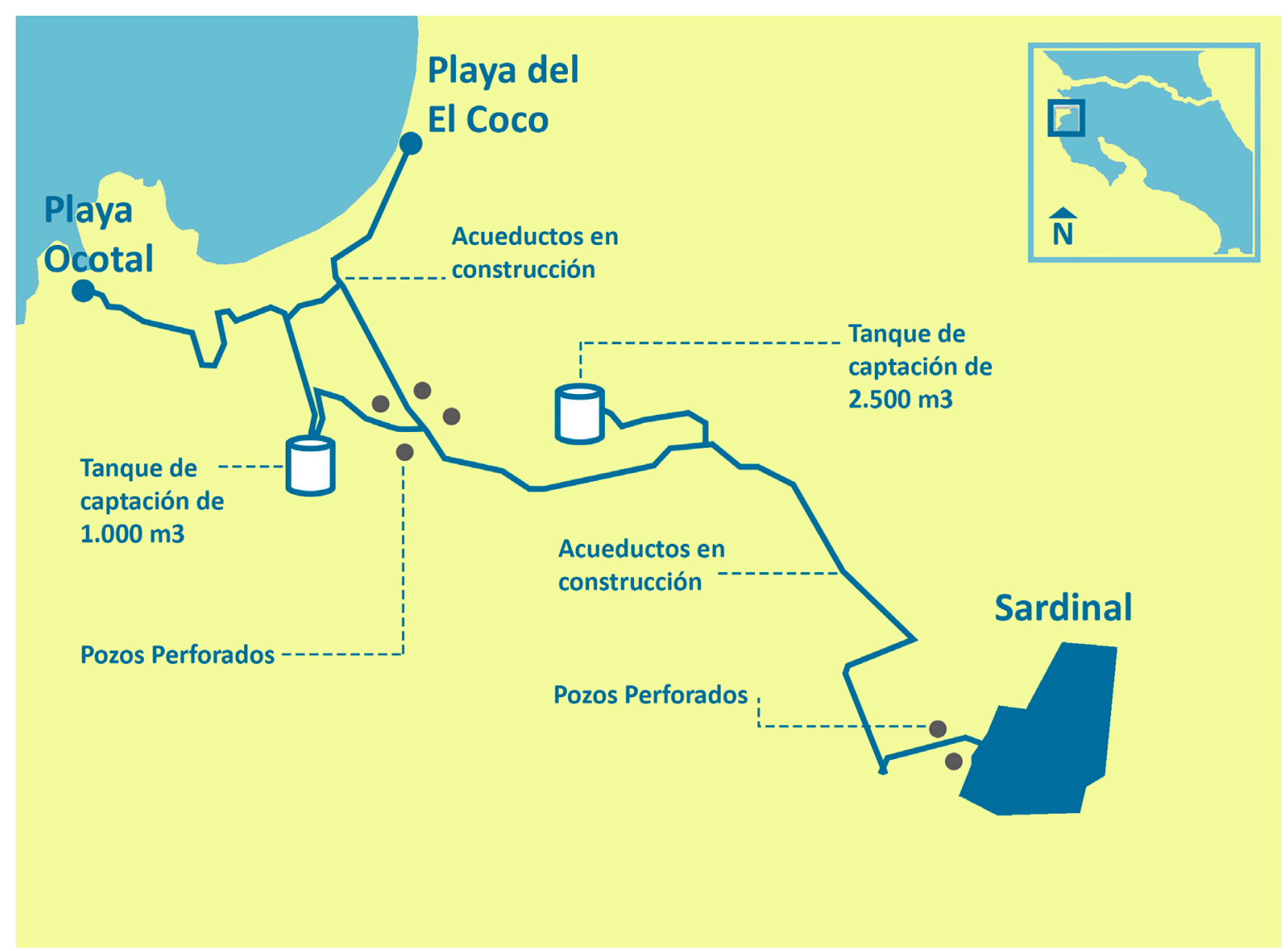


Ante esta resolución cabe preguntarse: ¿cómo la simple interpretación de un artículo (en este caso el Art. 38 de la LPU) permitió aprobar la construcción de un desarrollo turístico que requería $14 \mathrm{~km}$ de redes de infraestructura? Si bien es cierto el Art. 38 permite la construcción por parte del sector privado de infraestructura pública, ésta debe hacerse de manera excepcional, en los casos donde medie un claro interés público en la ampliación del sistema de alcantarillado y consecuente extracción de líquido. Inversión que debe realizarse con base en estudios técnicos que justifiquen las obras y que igualmente demuestren que no se pone en peligro el abastecimiento de las comunidades presentes y futuras (Cabrera \& Sánchez, 2009).

Por otra parte, las consecuencias que una ampliación de $14 \mathrm{~km}$ de infraestructuras tendría sobre el crecimiento urbano del cantón de Carrillo es también parte de las preocupaciones asociadas. Esta nueva infraestructura promovería el crecimiento descontrolado de la ciudad hacia las zonas turísticas abastecidas.

El Art. 38 presenta el agravante que permite al desarrollador que realiza la ampliación del servicio cobrar a los proyectos futuros por el usufructo de la infraestructura. En ese sentido, se promueve la expansión de la mancha urbana como forma de recuperar la inversión realizada.

Otro caso similar es el acontecido en el cantón de Santa Cruz, con la construcción de un acueducto de $16 \mathrm{~km}$ para abastecer de agua varios proyectos turísticos, desde el acuífero de Nimboyores hasta playa Conchal. Como en el caso anterior, las comunidades se opusieron y presentaron querellas ante las instancias de justicia alegando que se pone en riesgo el acuífero y el acceso al recurso hídrico por parte de las comunidades. La Sala Constitucional falló a favor de las comunidades.

En lo que respecta a planes reguladores costeros, se han dado casos donde las zonificaciones aprobadas han sido recurridas por amenazar zonas protegidas, o bien, intentar regular áreas que son competencia del Ministerio de Ambiente. Tal es el caso del plan regulador costero en el distrito de Cabo Velas, en el cantón de Santa Cruz, del sector norte de playa Ventanas al sector Sur de playa Langosta.

La zonificación establecida por el plan regulador fue llevada ante los tribunales, en 2008, por una organización conservacionista. Los recurrentes alegaron que dicho plan ponía en peligro la zona de anidación de la tortuga baula [figura 8]. En la acción de inconstitucionalidad presentada, se argumenta que la actividad derivada de hoteles, casas, condominios, restaurantes y demás infraestructura turística contraviene el objetivo de proteger y defender el proceso de anidación de la tortuga baula (Sala constitucional, resolución No 2008-008713).

En el por tanto de la resolución emitida por la Sala Constitucional, se declara con lugar la acción presentada y se anula el Reglamento de Zonificación del Distrito Cabo Velas dictado por el Concejo Municipal de Santa Cruz, alegando que la protección del desove de la tortuga baula es de importancia para la conservación de la especie y de la actividad turística y ecológica (Sala Constitucional, Resolución N. ${ }^{0}$ 2008-008713).

\section{Conclusiones}

Los impactos provocados por el turismo sobre los recursos naturales tienen un origen diverso y multicausal. La ausencia de planes reguladores actualizados y con variable ambiental, incluida en la gran mayoría del territorio de la región, es uno de los principales problemas detectados. Esta carencia es, en gran medida, producto de la debilidad de los gobiernos locales y la desarticulación normativa e institucional presente en el ordenamiento territorial.

Para los municipios que no cuentan con planificación urbana la interpretación de las normas nacionales representa una respuesta insuficiente en las labores de tramitación y control. Aunado a esto, la debilidad de los municipios de la región en las labores de planificación y fiscalización repercute directamente en el cumplimiento de la normativa ambiental. Las carencias de los gobiernos locales radican, básicamente, en la ausencia de recursos técnicos y humanos en los temas de planificación urbana y ordenamiento territorial. 


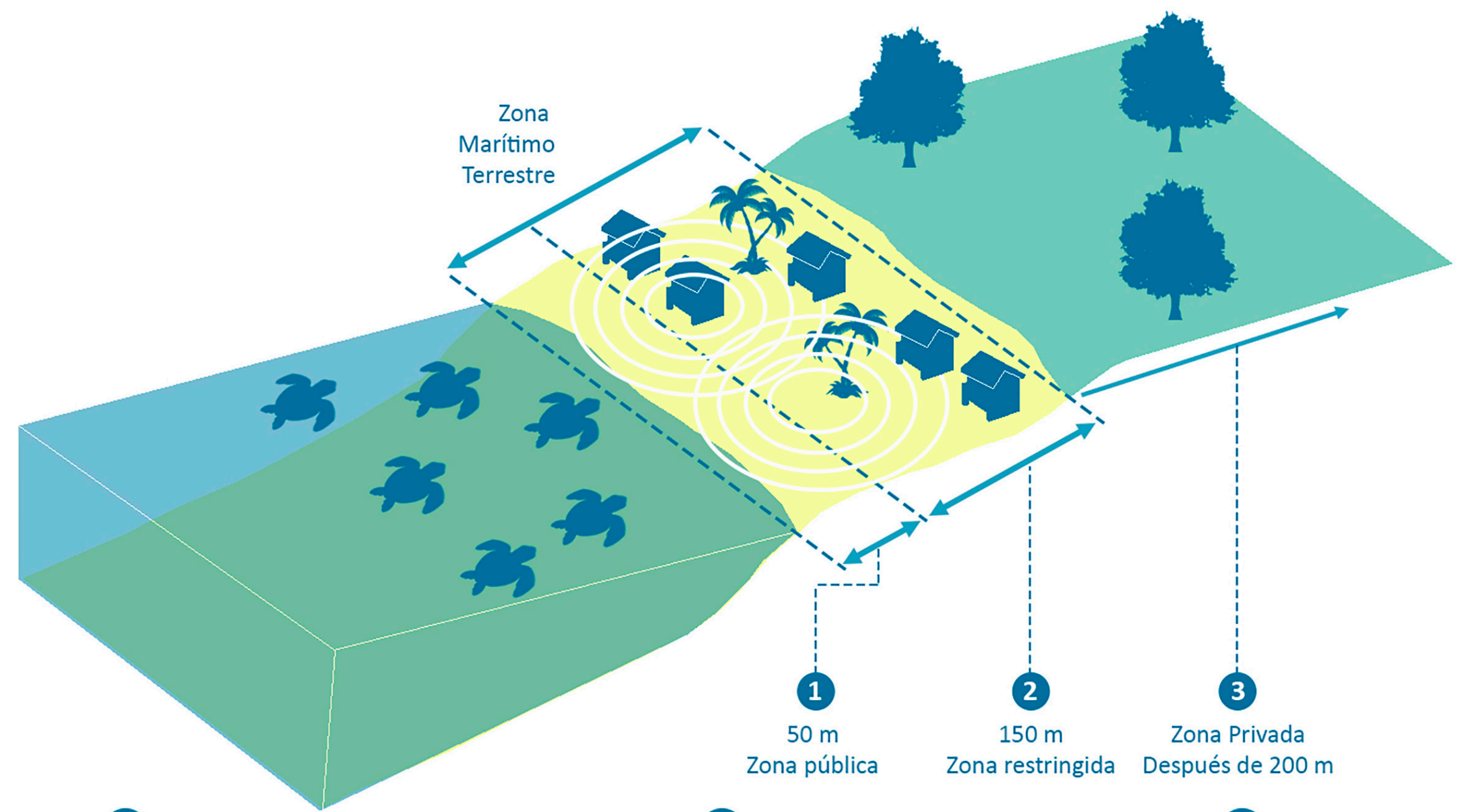

1

Propiedad del estado. 50 metros desde la línea de pleamar.
Propiedad del estado. Las municipalidades pueden otorgar concesiones según el plan regulador costero.

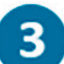

Terrenos privados. regulados según indique el plan regulador cantonal.
Figura 8. Detalle de lo establecido en la Ley de la Zona Marítimo Terrestre. Aunque la Ley de la Zona Marítimo Terrestre habilita la concesión en la zona restringida, en el caso de Cabo Velas la zonificación propuesta por el Plan Regulador debió ser cambiada para defender a la tortuga Baula de los efectos producto de hoteles, casas y restaurantes. Imagen de elaboración Propia.
La construcción es una actividad transversal al tema de planificación y desarrollo costero. Impacta en la medida en que se planifique y ordene el territorio ya que su crecimiento es un parámetro para identificar el perfil de los usos del territorio y su organización administrativa a nivel local (Cabrera \& Sánchez, 2009, p.66)

La inexistencia de normas territoriales de índole local, ha dado pie a interpretaciones de la normativa nacional que riñen con la defensa de los recursos naturales y las comunidades. Tal es el caso de los acuíferos Sardinal y Nimboyores. Adicionalmente, la aplicación de normas nacionales reduce el análisis ambiental de los proyectos turísticos a estudios puntuales para cada caso. Este vacío solo es subsanable con la presencia de planes reguladores que cumplan con el debido proceso de introducción de la variable ambiental.

Es claro que la llegada de la industria turística y el establecimiento de enclaves turísticos no ha estado acompañada con una política adecuada de fortalecimiento e impulso a la planificación urbana. Esto queda en evidencia cuando se constata el escaso alcance de los planes reguladores cantonales aprobados y su nivel de desactualización. En el caso de la zona marítimo terrestre, existe una mayor cantidad de planes reguladores aprobados. Sin embargo, estos planes se limitan, únicamente, a la franja de litoral de $200 \mathrm{~m}$ desde la línea de pleamar, lo que no garantiza la conservación de los recursos naturales fuera de esta zona.

El crecimiento de la actividad turística en la región Chorotega no ha sido acompañado con una política de actualización normativa y fortalecimiento del ordenamiento territorial. Esta condición genera que la región intente regular una industria en constante movimiento, con normativas que, en muchos casos, no responden al contexto social y ambiental actual. Tal es el caso de la Ley de Aguas de 1942 y la Ley de Planificación Urbana de 1967.

Con la llegada del turismo a Guanacaste en la década 1990 y su boom en la siguiente década, ocurrió un crecimiento sin planificación ni ordenamiento territorial. Se otorgó una gran cantidad de permisos de construcción, con una mirada cortoplacista, sin sopesar, entre otras cosas, un posible desabastecimiento de agua (Navas \& Cuvi, 2015, p. 116) 
Por otra parte, aunque el municipio cuente con la normativa requerida para la planificación, han existido casos donde esta normativa no esa diseñada para tomar en cuenta las características específicas de los diferentes recursos naturales de los cuales están haciendo uso.

Lo acontecido en el caso del plan regulador de Cabo Velas revela que este tipo de normativa no toma en cuenta los procesos eco sistémicos de interés turístico. En este sentido, los límites, parámetros de uso, densidad de ocupación y otros aspectos propios de la actividad turística, no se encuentran personalizados a los recursos ambientales que el turista disfruta. Estos parámetros no pueden ser homogéneos en todos los casos, dado que las dinámicas ambientales varían según el recurso turístico. La forma en que la tortuga baula anida no es equiparable al ámbito de hogar del manigordo, 0 bien, al uso del hábitat que realiza el venado cola blanca. Estas particularidades que responden directamente a la localización y el tipo de actividad turística, están ausentes en la normativa para la regulación de los proyectos turísticos.

La introducción de la variable ambiental en los planes reguladores -tanto cantonales como costeros- debe incluir los procesos naturales de escala regional o subregional que puedan ser afectados por la actividad turística inmobiliaria. La metodología establecida para tal efecto permite hacer este análisis. Sin embargo, en algunos casos, esto no ha sido tomado en cuenta.

Otro aspecto que influye en la poca efectividad en la tramitación, es la carencia de información sobre el estado de los recursos naturales. En el caso del recurso hídrico la información sobre la capacidad de acuíferos y ríos es limitada, aspecto que provoca su explotación de forma no planificada y desproporcionada (Tribunal Centroamericano del Agua, 2014).

La presencia de muchas instituciones con competencias en los procesos de tramitación y planificación, complica y reduce la efectividad de los procesos de control.

Desde el punto de vista del recurso hídrico, es posible identificar muchas instituciones que tienen participación en el manejo y gestión del recurso. Esta condición provoca que no exista un único responsable, lo que termina generando brechas y diluyendo la responsabilidad técnica en la planificación y control (Guzmán y Calvo, 2012).

El choque entre la política económica y la política ambiental de Costa Rica, así como las debilidades en el marco jurídico e institucional del Estado, tuvieron como consecuencia respuestas contradictorias por parte de las instituciones estatales a la expansión del turismo residencial en Guanacaste y a su impacto ambiental y social negativo (Barrantes, 2013, p. 252)

Ante este panorama se plantean una serie de acciones para revertir la situación:

Renovar los instrumentos de planificación territorial, fortaleciendo las capacidades de gestión y control de los municipios. Las municipalidades deben estar en capacidad de elaborar y poner en práctica planes reguladores que consideren las particularidades de cada territorio e incluyan el componente ambiental. La participación civil en la planificación territorial es un insumo importante para la veracidad y correcta aplicación posterior.

La norma urbanística recoge la mayoría de condiciones que permiten la ocupación de zonas frágiles y dificultan la conservación de los recursos naturales. Adicionalmente, es la normativa de mayor antigüedad que incide en el establecimiento de proyectos turísticos. Su actualización es necesaria para eliminar los vacíos detectados, facilitar los procesos de control y homologar conceptos con otras leyes.

En el caso específico de los planes reguladores, su elaboración debe tomar en cuenta los procesos eco sistémicos que dan pie a la inversión en turismo. Estos planes deben trascender de las zonificaciones rígidas para responder específicamente a las dinámicas naturales de la flora y la fauna local. Esta consideración evitaría que una zonificación puntual afecte procesos ecosistémicos de escala superior.

La falta de información sobre el estado real de los recursos naturales en la región es una carencia importante. El fortalecimiento de la investigación sobre la capacidad de carga de los diferentes recursos naturales disminuiría la incertidumbre y minimizaría el otorgamiento inadecuado de permisos de construcción. 


\section{Referencias bibliográficas}

Barrantes Reynolds, M. P. (2013). "Costa rica, sin ingredientes artificiales": El rol del estado en la expansión del turismo residencial en las zonas costeras. Anuario De Estudios Centroamericanos, (39), 233-261. Recuperado desde: https://dialnet. unirioja.es/descarga/articulo/5075716.pdf

Cabrera, J., \& Sánchez, S. (2009). Marco legal y estructura institucional del desarrollo turístico e inmobiliario en la costa pacífica de costa rica. Informe Final. Preparado por the Center for Responsible Travel.

Decreto 32967. Manual de instrumentos técnicos para el proceso de evaluación del Impacto ambiental. Diario oficial La Gaceta No85, San José de Costa Rica. 4 de mayo del 2006.

Guzmán-Arias, I., \& Calvo-Alvarado, J. C. (2012). Recursos hídricos de la cuenca alta del río tempisque, costa rica (nota técnica). Tecnología En Marcha, 25(4), 63-70. Recuperado desde: https://dialnet.unirioja.es/descarga/articulo/4835664.pdf

Honey, M., Vargas, E., \& Durham, W. (2010). Impacto del turismo relacionado con el desarrollo en la costa Pacífica de Costa Rica (Informe ejecutivo). Center for Responsible Travel. San José, Costa Rica.

Honey, Martha; Krantz, David. (2007). Global Trends in Coastal Tourism. 8/04/2016, de Center on Ecotourism and Sustainable Development. Recuperado desde: http:// www.responsibletravel.org/resources/documents/reports/global_trends_in_coastal_tourism_by_cesd_jan_08_lr.pdf

Ley № 276. Ley de Aguas. Asamblea Legislativa, San José de Costa Rica, 27 de agosto de 1942.

Ley No 7794. Ley de Biodiversidad. Diario Oficial La Gaceta N²6, San José de Costa Rica. 06 de febrero de 1998.

Ley $N^{0}$ 7788. Ley de Concesión y Operación de Marinas y Atracaderos Turísticos. Diario Oficial La Gaceta N0101, San José de Costa Rica. 27 de mayo de 1998.

Ley № 833. Ley de Construcciones. Asamblea Legislativa, San José de Costa Rica, 2 de noviembre de 1949 .

Ley № 7575. Ley Forestal. Diario Oficial La Gaceta №72, San José de Costa Rica. 16 de abril de 1996.

Ley № 7554. Ley Orgánica del Ambiente. Diario Oficial La Gaceta No215, San José de Costa Rica. 13 de noviembre de 1995.

Ley No 1917. Ley Orgánica del Instituto Costarricense de Turismo. Diario Oficial La Gaceta N0175, San José de Costa Rica, 9 de agosto de 1955.

Ley No 4240. Ley de Planificación Urbana. Asamblea Legislativa, San José de Costa Rica. 15 de noviembre de 1968.

Ley No 6043. Ley sobre la Zona Marítimo Terrestre. Diario Oficial La Gaceta N 52, San José de Costa Rica, 16 de marzo de 1977.

Ley № 7779 . Ley de Uso, Conservación y Manejo de Suelos. Diario Oficial La Gaceta No97, San José de Costa Rica. 21 de mayo de 1998.

Maxera, E. C. (2008). Problemas graves en las costas de Costa Rica. Revista De Ciencias Jurídicas, (120).

Navarro Cerdas, S. (2014). Geopolítica en una "periferia del placer". colonialidad turística en costa rica. Revista De Ciencias Sociales, (145), 45-60.

Navas, G., \& Cuvi, N. (2015). Análisis de un conflicto socioambiental por agua y turismo en sardinal, costa rica. Revista De Ciencias Sociales, (150), 109-124. Recuperado desde: https://dialnet.unirioja.es/servlet/articulo?codigo=5328319\&orden=0\&info=link

Ramírez Cover, A. (2007). Conflictos socio-ambientales y recursos hídricos en Guanacaste. una descripción desde el cambio en el estilo de desarrollo (1997- 
2006). Anuario De Estudios Centroamericanos, (33), 359-385. Recuperado desde: https://dialnet.unirioja.es/descarga/articulo/5075784.pdf

Resolución Nº 2008-008713. Acción de inconstitucionalidad contra el reglamento de zonificación del distrito de Cabo Velas.

Resolución N² 2006-013462. Recurso de amparo contra el Ministerio de Ambiente y otros, por obras de construcción en playa Hermosa de Carrillo.

Román, Marcela. (2006). Desarrollo Turístico e inmobiliario costero, preocupaciones ambientales. 19/02/2016, de Programa Estado de la Nación Sitio Web: http:// www.estadonacion.or.cr/files/biblioteca_virtual/013/Desarrollo_costero_preocupaciones_ambientales.pdf

Sala Constitucional de la Corte Suprema de Justicia. San José de Costa Rica. Resolución No 2009-000262.

The Authority on World Travel and Tourism. (2015). Travel and Tourism. Economic Impact 2015 Costa Rica. 11/04/2016, de World, Travel and Tourism Council sitio web: http://www.wttc.org/-/media/files/reports/economic\%20impact\%20research/ countries\%202016/costarica2016.pdf

Tribunal Centroamericano del Agua. (2004). Resumen Audiencia de Juzgamiento; Costa Rica eventual explotación del acuífero Nimboyores en Santa Cruz, Guanacaste. 11/04/16, de Tribunal Latinoamericano del Agua Sitio web: http://tragua.com/ wp-content/uploads/2012/04/caso_acuifero_nimboyores.pdf

Van Noorloos, Femke. (2013). ¿Un Lugar en el Sol para quién?. 8/02/2016, de Alba sud, investigación y comunicación para el desarrollo Sitio web: http://www.albasud.org/publ/docs/58.pdf 